Recepción: 04/09/2018

Aceptación: 11/11/2018

Publicación:03/12/2018

Ciencias sociales y políticas

Artículo científico

\title{
El marketing político y su impacto en la campaña electoral de los precandidatos a la alcaldía de Manta - Ecuador
}

\section{Political marketing and its impact on the electoral campaign of pre-candidates for mayor of Manta - Ecuador}

\section{Marketing político e seu impacto na campanha eleitoral de pré-candidatos a prefeito de Manta - Equador}

Johnny Edison Ponce-Andrade ${ }^{\mathrm{I}}$

johnny.ponce@uleam.edu.ec

Anthony Rolando Zambrano-Zambrano II

anrolzam@hotmail.com

Ana Elena Arias-Arias III

ana.arias@uleam.edu.ec
Ulises Federico Mero-Chávez IV ulises.mero@ hotmail.com

Wagner Oswaldo Villafuerte-Muñoz ${ }^{\mathrm{V}}$

wagnermatador24@hotmail.com

Edison Aroldo Gracia-Panta VI edison.gracia@uleam.edu.ec

Correspondencia: johnny.ponce@uleam.edu.ec

I. Doctor en Ciencias Administrativas, Diploma Superior en Educación Universitaria por Competencias, Magíster en Dirección Estratégica de Marketing y Ventas, Especialista en Gerencia Estratégica de la Comunicación Organizacional, Ingeniero Comercial, Docente Universidad Laica Eloy Alfaro de Manabí, Manta, Ecuador.

II. Estudiante de la Universidad Laica Eloy Alfaro de Manabí, Manta, Ecuador.

III. Magíster en Marketing, Licenciada en Secretariado Ejecutivo, Especialista en Diseño Curricular por Competencias, Especialista en Gerencia Estratégica de la Comunicación Organizacional, Licenciado en Ciencias de la Comunicación Especialidad: Relaciones Publicas, Técnico en Relaciones Publicas, Docente Universidad Laica Eloy Alfaro de Manabí, Manta, Ecuador.

IV. Máster Universitario en Dirección y Gestión de Recursos Humanos, Ingeniero Comercial, Docente de la Universidad Laica Eloy Alfaro de Manabí, Manta, Ecuador.

V. Magíster en Enseñanza del Idioma Inglés, Diploma Superior en Gestión Directiva, Licenciado en Ciencias de la Educación Mención en Inglés, Docente Universidad Laica Eloy Alfaro de Manabí, Manta, Ecuador.

VI. Magíster en Educación y Desarrollo Social, Abogado de los Juzgados y Tribunales de la Republica, Licenciado en Ciencias de la Educación Especialización Físico Matemáticas, Profesor de Segunda Enseñanza Especialidad Físico Matemático , Docente Universidad Laica Eloy Alfaro de Manabí, Manta, Ecuador.

http://polodelconocimiento.com/ojs/index.php/es 


\section{Resumen}

La base fundamental para entender las concepciones teóricas, es en la práctica transponer conceptos sobre realidades. La temática abordada es probablemente uno de los ejes más significativos del pragmatismo mercadotécnico, por medio del "politing", conocido como marketing político integrado, el cual se convierte en un sistema heterogéneo, de teorías, praxis, métodos y técnicas que ayudan a las organizaciones políticas a entender el mercado elector. Como una actividad que se deriva de la ciencia política, la sociología y la comunicación, se sostiene en la investigación, la estrategia y la comunicación política. Se escoge entonces como escenario de estudio a la ciudad de Manta, provincia de Manabí, República del Ecuador, la cual se apresta a vivir un proceso eleccionario para su gobierno seccional. Se utilizan las variables Marketing Político y Campaña electoral, por estar íntimamente relacionadas, tal como lo afirman Josehp Napolitan y Daniel Skibel (2015). Existe el propósito de conocer en esta era de incesantes avances tecnológicos, como estas nuevas herramientas, al servicio de un nuevo concepto "marketing político digital", son utilizadas por los precandidatos a burgomaestre del cantón Manta, por ello el objetivo general de esta investigación es "determinar de qué manera el Marketing Político impacta en la campaña electoral de los precandidatos a la Alcaldía de Manta Ecuador". El método aplicado es lógico inductivo completo, de carácter cuali cuantitativo. Se aplicaron 11 encuestas a postulantes a la Alcaldía, es un esquema de preguntas en escala de Likert, que fueron procesadas en el software estadístico SPSS 21, para validar la hipótesis de estudio.

Palabras clave: Marketing; campaña electoral; votante; organización política; precandidato.

\section{Abstract}

The fundamental basis for understanding theoretical conceptions is, in practice, to cross concepts about realities. The topic addressed is probably one of the most significant axes of marketing pragmatism, through "politing", known as political marketing, which becomes a heterogeneous system of theories, praxis, methods and techniques that help the political organizations understand the voter market. As an activity that derives from political science, sociology and communication, it is sustained in research, strategy and political communication. The city of Manta province of Manana, Republic of Ecuador, is chosen as a study scenario, which is preparing to live an election process for its sectional government. The variables Political 
Marketing and Electoral Campaign are used, because they are intimately related, as Josehp Napolitan and Daniel Skibel (2015). There is the purpose of knowing in this era of incessant technological advances, as these new tools, at the service of a new concept "digital political marketing" are used by the precandidates for mayor of the Manta canton, for that reason the general objective of this research is " Determine how political marketing impacts the electoral campaign of the mayoral precandidates in Manta - Ecuador". The applied method is a complete inductive logic, of a qualitative and quantitative nature. 11 surveys were applied to the applicants to the mayor's office, it is a questionnaire scheme in Likert scale, which were processed in the statistical software SPSS 21, to validate the study hypothesis.

Keywords: Marketing; electoral campaign; voter; political organization; precandidate.

\section{Resumo}

A base fundamental para a compreensão de concepções teóricas é, na prática, transpor conceitos sobre realidades. Os temas abordados é provavelmente um dos mais significativos pragmatismo centros comerciante, através do "Politing" conhecido como marketing político integrado, que se torna um heterogêneos sistema, teoria, prática, métodos e técnicas que ajudam as organizações Políticas para entender o mercado eleitoral. Como uma atividade que deriva da ciência política, sociologia e comunicação, ela é sustentada em pesquisa, estratégia e comunicação política. A cidade de Manta, província de Manabí, República do Equador, é escolhida como cenário de estudo, que se prepara para viver um processo eleitoral para seu governo seccional. As variáveis Marketing Político e Campanha Eleitoral são utilizadas, pois estão intimamente relacionadas, conforme afirmado por Josehp Napolitan e Daniel Skibel (2015). Destina-se a conhecer nesta época de incessantes avanços tecnológicos, como vai você novas ferramentas a serviço de um novo conceito de "marketing político digitais" são utilizados pelos candidatos a prefeito do cantão Manta, de modo que o objetivo geral da pesquisa é "Determinar como o marketing político impacta a campanha eleitoral dos candidatos ao prefeito de Manta - Equador". O método aplicado é uma lógica indutiva completa, de natureza qualitativa. 11 inquéritos candidatos a prefeito foram aplicados, é um esquema de perguntas de escala de Likert, que foram processados em SPSS 21 Statistical Software para validar a hipótese do estudo.

Palavras-chave: Marketing; propaganda eleitoral; eleitor organização política; pré-candidato 


\section{Introducción}

Es probable que Manta sea hoy uno de los centros más hegemónicos de la economía nacional, su desarrollo ha ido de la mano con el de la provincia; genera una contribución del 7 \% del PIB de Manabí, siendo el cantón de la trasformación productiva, pues su tejido empresarial permite generar la mayor cantidad de plazas de trabajo a nivel provincial y en buena parte del país. Por ello quien llegue a ocupar el sillón de la máxima personería local, no solo debe tener capacidades cognoscentes, sino un alto sentido de responsabilidad, frente a un reto solo de valientes, pues el futuro de la noble ciudad cuna de pescadores, artesanos y entusiastas emprendedores, se la delegara a quien goce del favor y la confianza ciudadana en el próximo proceso eleccionario.

Esta ciudad en camino de un galopante desarrollo, es también una urbe universitaria, donde el nivel sociocultural de sus habitantes es uno de los más desdoblados del país, por ello la tarea de quienes pretender alcanzar el cargo de "burgomaestre" tiene que acompañar a sus capacidades un buen diseño de campana electoral, mismo que en esta vertiginosa corriente de avances tecnológicos, puede marcar el elemento diferenciador para lograr el triunfo, en tanto y en cuanto se apropien de estos recursos, puesto que el electorado cada vez es más inteligente para decidir su voto, y no basta con un buen discurso ni una cara agraciada. Es aquí donde aparece el Marketing Político, que ha criterio de Avraham Shama, "es el proceso mediante el cual los candidatos políticos y las ideas son dirigidas a los votantes en orden de satisfacer sus necesidades políticas y ganar su apoyo para apuntalar al candidato y sus ideas". Así también El Doctor Bruce I. Newman, profesor de la Universidad DePaul en Chicago, lo define como "la aplicación de principios de mercadotecnia y procedimientos en las campañas políticas de varios individuos y organizaciones. Los procesos involucrados incluyen el análisis, desarrollo, ejecución y gerencia de campañas estratégicas de candidatos, partidos políticos, gobiernos, lobistas y grupos interesados en influenciar la opinión pública, dar avance a sus propias ideologías, ganar elecciones y hacer legislaciones en respuesta a las necesidades y requerimientos de personas selectas".

Entonces, ¿cuán beneficioso podría resultar para un candidato y su organización política aplicar el Marketing Político y más el integrado "Politing", para dosificar sus estrategias?, es lo que se trató de descubrir en el presente estudio, toda vez que lo que se apreció a simple vista, es la existencia de una descolorida propuesta, que no conmueve al electorado mantense, a escasos tres 
meses de las elecciones seccionales, siendo el gran problema detectado, el cual llena de desconcierto al candidato y al elector. Por ello esta investigación, a más de tener planteada ya su hipótesis, también lo hace a nivel de objetivo, por tanto, se cumple con una estructura metodológica-científica, que justifica plenamente el abordaje del tema.

\section{Desarrollo}

Para darle el sustento teórico - científico, a una de las temáticas muy poco analizadas en el medio, se han estudiados varios enfoques teóricos sobre la Mercadotecnia Política, dando énfasis a sus beneficios y aportes.

Wojciech Cwalina, Andrzej Falkoski y Bruce I. Newman, en el libro Political Marketing: Theoretical and Strategic Foudations, coinciden en la definición de que el Marketing Político "es la integración de propuestas que permiten tener un entendimiento del comportamiento humano", que es la base para lograr una buena campaña de marketing político, pues se hace “énfasis en los mecanismos psicológicos del comportamiento del votante" y que "no se limita solo a los periodos de elecciones y campañas, pues ya no hay diferencia entre la elección y el resto del calendario político". Mantener la estrategia durante toda la temporada, construye confianza, credibilidad y legitimización. El marketing político "es una disciplina, el estudio de procesos e intercambios entre entidades políticas y su ambiente, con referencia particular del posicionamiento de comunicaciones en distintas entidades".

Así, la esencia de esta disciplina, busca estrechar el nivel de relación entre el candidato y los votantes, para ello la comunicación y las formas de establecerlas, le permite al postulante construir una imagen consistente, que inspire confianza y seguridad, a fin de encausar la simpatía y aceptación del mercado electoral. Pero como todo ejercicio profesional, los principios deontológicos son fundamentales, y el marketing político debe manejarse de manera responsable, y la mejor forma de hacerlo es inquiriendo las necesidades de la sociedad, como también educando a los electores sobre el escenario político en torno a cada ciudadano, incentivando a su participación, con una fluida información antes y mucho más en el mandato, en caso de alcanzar el triunfo electoral.

Ahora, que necesita un candidato y su organización política para encaminar una campaña exitosa, lo primero es: 

1. Analizar el mercado electoral, el mismo que ayudara a comprender lo que mueve a las personas, que las hacer resistir a la aceptación del candidato, que se necesita para construir su creencia en un proceso electoral y sus postulantes. Es justamente en esta tarea donde se obtienen respuestas del mercado objetivo (votantes), y para ello se hace uso de herramientas de la investigación, como las encuestas, sondeos de opinión, tracking poll, entre otros elementos para levantar información. Aquí ya la tecnología brinda un importante soporte, por el uso de dispositivos tecnológicos que permiten el procesamiento de entrada y salida de información, siendo las redes sociales y el internet, un valiosísimo instrumento para mantenerse contactado con el electorado.

2. El segundo aspecto importante que enseña el marketing, es la creación de una marca política, pues de la misma forma que en los negocios, los políticos también necesitan llevar una "marca", puesto que la misma le da una impronta de identidad al político, siendo esta la que denote e inspire los ideales políticos de la organización y/o candidato. Los perfiles de los candidatos deben ser estudiados a partir de la planeación de una imagen que se proyectara a la sociedad. Ejemplarizando, ciertos candidatos son reconocidos por sus electores como representantes de un sector o gremio, como salud, educación, deportes, economía, comercio, etc.; en resumen, el marketing ayudara a construir la estrategia de branding al político.

3. Aprovechando los recursos tecnológicos, para lograr una buena fuerza política y ejecutar sus proyectos, el candidato debe "construir una comunidad", y para eso las redes sociales permiten una popularización más rápida del político, ya no es suficiente el activismo y la militancia política, hoy se requiere de los elementos en mención, y para aquello es necesario contar con un asesor experto en la materia.

4. Es necesaria una inversión en contenidos, produciendo material de calidad, procurando educar al electorado, esto contribuirá a la propagación de la imagen del candidato, dado que buenos contenidos tienen la tendencia de ser compartidos en las redes sociales, con un alcanzase incalculable. Dichos contenidos, generan "leads", buscando atraer personas, conducidos a una comunidad que luego se convierten en apoyadores.

5. La potenciación de presencia en las redes sociales, mantiene contactado al elector con el candidato, donde el intercambio de información es generador de nuevas ideas y proyectos, 
aprovechando el político para mantener informada a la comunidad sobre sus acciones y movimientos.

Hay muchos ejemplos del éxito que han tenido varios candidatos a nivel global aplicando el Marketing Político Integrado y digital, tales como Barack Obama y Bernia Sanders, quienes no solo fortalecieron sus candidaturas, sino que lograron hacer una recaudación de fondos de apoyo para sus campañas sin precedentes históricos.

\section{Objetivo de la investigación}

Determinar de qué manera el Marketing Político impacta en la campaña electoral de los precandidatos a la Alcaldía de Manta - Ecuador.

\section{Hipótesis de la investigación}

Toda investigación debe plantear una hipótesis, por ello se delineo el siguiente supuesto: El Marketing Político impacta en la campaña electoral de los precandidatos a la Alcaldía de Manta Ecuador.

\section{Metodología}

La metodología de investigacion es lógica inductiva completa, a través de hipótesis; en el presente estudio fue necesario aplicar la investigación exploratoria, descriptiva y explicativa, así lo que se pretende en primer orden es explorar el tema para saber más de él, después se detallan las variables involucradas, subsiguientemente se explica el impacto de una variable sobre la otra en términos de causalidad.

\section{Diseño de la investigación (mediante encuesta)}

Este es un instrumento efectivo de medición para el levantamiento de información de corte cuantitativo, mediante una encuesta con preguntas en escala de Likert, en cuyo diseño de carácter no experimental y no transversal, tal como lo determinan Campbell y Stanley (1978), "se toma la información en un momento determinado del tiempo, para obtener datos relevantes y sustanciales de la población de estudio" (p.40). Esta técnica permite conocer el problema del sector desde la óptica del candidato y su organización política.

\section{Unidad de análisis}

El estudio se realizó en la ciudad de Manta a 11 precandidatos a la Alcaldía del Cantón; la fuente informativa son medios de prensa, bases de las organizaciones políticas y el Consejo Nacional Electoral de Manabí (CNE). 


\section{Tamaño de la muestra}

El tamaño de la muestra está definido de acuerdo al tipo de muestreo, que en este caso es por conveniencia, dado que los individuos empleados en la investigación son de fácil disponibilidad y acceso, además por los tiempos del estudio existe una gran facilidad operativa y bajos costos. Para darle sustento teórico al enunciado se cita a John W. Creswell (2008) quien lo define "como un procedimiento de muestreo cuantitativo, en el que el investigador selecciona a los participantes, ya que están dispuestos y disponibles para ser estudiados”.

\section{Técnicas de recolección de datos}

Una vez determinada la muestra a ser investigada, se procedió a la aplicación de la encuesta, Se corrieron 11 encuestas en Manta, en un sistema directo, cuyo instrumento original está conformado por 26 ítems, relacionadas con las variables, evaluadas por medio de escala de Likert, con calificación de hasta 5 puntos, en el siguiente orden: Nada (equivale a 1), Poco (equivale a 2), Medianamente (equivale a 3), Mucho (equivale a 4), Ampliamente (equivale a 5). A través de la aplicación del software SPSS 21, se procesó el banco de preguntas sobre las variables Marketing Político y Campaña Electoral (26 ítems en 11 encuestas), donde primero se realizó un análisis de fiabilidad para evaluar la confiabilidad del instrumento de medición por medio del Alfa de Cronbach. Este coeficiente establece que mientras el valor calculado sea más cercano a uno (1), mayor será la confiabilidad del instrumento. El resultado se detalla en la siguiente tabla.

\section{Tabla 1 Fiabilidad Alfa de Cronbach Estadísticos de fiabilidad}

\begin{tabular}{cc}
\hline Alfa de Cronbach & N de elementos \\
\hline, 991 & 26 \\
\hline
\end{tabular}

Fuente: Sistema SPSS 21.

La tabla 1 muestra la fiabilidad Alfa de Cronbach de 0,991 por consistencia para los 26 ítems, el resultado de este coeficiente nos indica una excelente fiabilidad.

\section{Resultados}

\section{Prueba de hipótesis}

Una vez que se corrieron las encuestas con opciones de tipo Likert, con cinco categorías de respuestas, en escalas nominales. Se realizó la prueba no paramétrica bivariado, mediante el 
análisis de correlación de Rho de Spearman para la comprobación de la hipótesis en el programa estadístico SPSS 21. Para la interpretación de los resultados es importante detallar el baremo de coeficiente de correlación, el cual estipula lo siguiente: Si el coeficiente esta entre 0 y 0,2 entonces la correlación es mínima; si esta entre 0,2 y 0,4 es baja; si esta entre 0,4 y 0,6 es moderada; si es de 0,6 a 0,8 es buena, y si encuentra entre 0,8 a 1, es una correlación muy buena.

\section{Tabla 2}

\section{Correlación de Spearman, Hipótesis General}

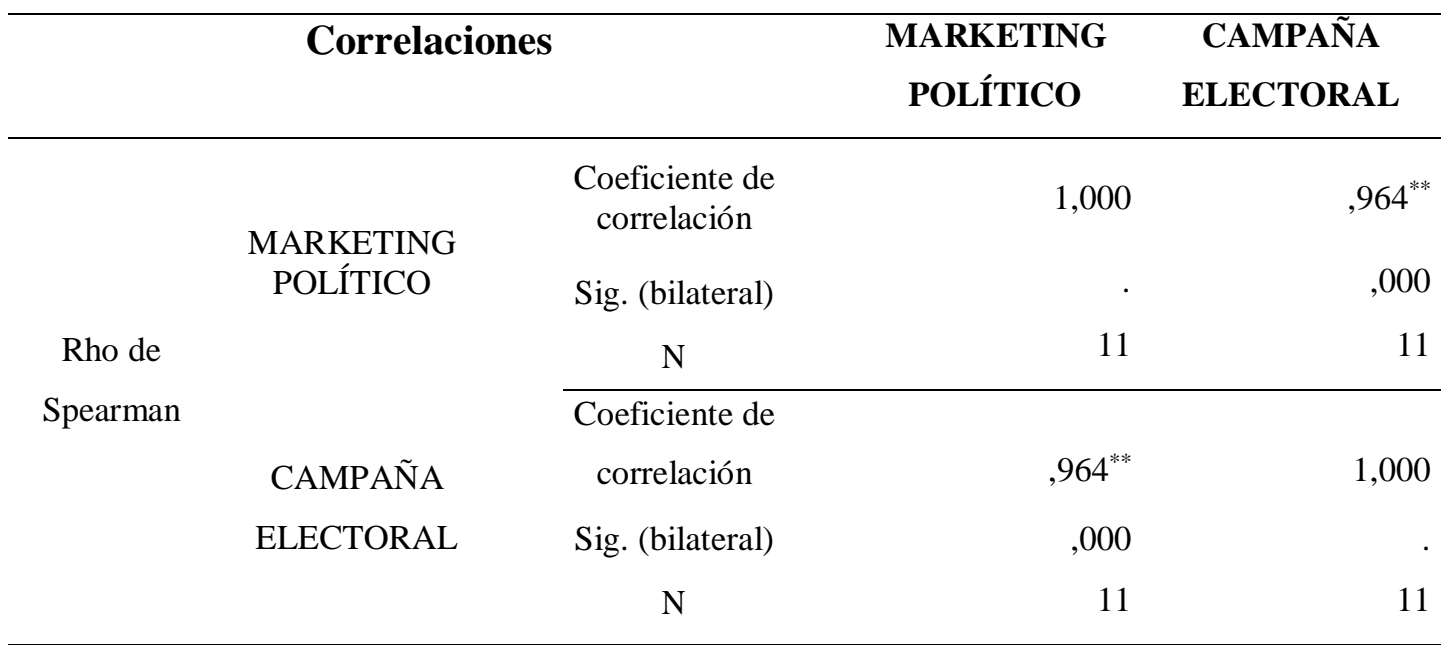

**. La correlación es significativa al nivel 0,01 (bilateral).

Fuente: Fuente: Sistema SPSS 21.

Analizando la tabla 2, procesada en el programa SPSS 21, se pudo determinar que existe un nivel de correlación alto o muy bueno (0,964), dado de que acuerdo al baremo está muy cercano a 1 . Esto indica que existe una correlación positiva, significando que, a mayor nivel de aplicación de Marketing Político, mayor será el resultado de la Campaña Electoral, por lo tanto, el escogimiento de las variables es acertada. Respecto al nivel se significación, se aprecia que es de 0,000, lo que reafirma el cumplimiento de la condición de que el grado de significación sea menor a 0,05 , incluso menor a 0,01 , lo que muestra que la correlación establecida es cierta, de tal manera que se rechaza la hipótesis nula y se acepta la hipótesis alternativa. 
Tabla 3 ¿Considera de importante el marketing político en una campaña electoral?

\begin{tabular}{|c|c|c|c|c|}
\hline & Frecuencia & Porcentaje & $\begin{array}{c}\text { Porcentaje } \\
\text { valido }\end{array}$ & $\begin{array}{l}\text { Porcentaje } \\
\text { acumulado }\end{array}$ \\
\hline Valido $\quad$ AMPLIAMENTE & 4 & 36,4 & 36,4 & 36,4 \\
\hline MUCHO & 2 & 18,1 & 18,1 & 54,5 \\
\hline MEDIANAMENTE & 1 & 9.1 & 9,1 & 63,6 \\
\hline $\mathrm{POCO}$ & 1 & 9,1 & 9,1 & 72,7 \\
\hline NADA & 3 & 27,3 & 27,3 & \\
\hline Total & 11 & 100 & 100 & \\
\hline
\end{tabular}

Fuente: Sistema SPSS 21.

De acuerdo a los resultados de la tabla 3, se evidencia que un $36,4 \%$ de los precandidatos encuestados consideran ampliamente importante al Marketing Político en una campaña electoral; un $18,1 \%$ indicaron que es de mucha importancia; un $9.1 \%$ de mediana importancia; otro $9.1 \%$ de poca importancia y finalmente un $27,3 \%$ expresa nada de importancia.

\section{Tabla 4}

\section{¿Utiliza publicidad televisiva para promover su campaña electoral?}

\begin{tabular}{ccccc}
\hline & Frecuencia & Porcentaje & $\begin{array}{c}\text { Porcentaje } \\
\text { valido }\end{array}$ & $\begin{array}{c}\text { Porcentaje } \\
\text { acumulado }\end{array}$ \\
\hline Valido AMPLIAMENTE & 1 & 9.1 & 9.1 & 9,1 \\
MUCHO & 1 & 9,1 & 9,1 & 18,2 \\
MEDIANAMENTE & 0 & 0,0 & 0,0 & 18,2 \\
POCO & 1 & 9,1 & 9,1 & 27,3 \\
NADA & 8 & 72,7 & 72,7 & \\
Total & 11 & 100 & 100 & \\
\hline
\end{tabular}

Fuente: Sistema SPSS 21.

La tabla 4 indica que un 9,1\% de los encuestados utilizan de manera amplia la publicidad televisiva para la promoción de su campaña electoral; un 9,1\% dijo que hay mucho uso; medianamente no obtuvo respuestas; otro $9.1 \%$ poco y finalmente el $72,7 \%$ expresaron nada de uso de medios televisivos por sus altos costos. 
Tabla 5

¿Utiliza publicidad radial para promover su campaña electoral?

\begin{tabular}{llcccc}
\hline & Frecuencia & Porcentaje & $\begin{array}{c}\text { Porcentaje } \\
\text { valido }\end{array}$ & $\begin{array}{c}\text { Porcentaje } \\
\text { acumulado }\end{array}$ \\
\hline Valido & AMPLIAMENTE & 7 & 63,7 & 63,7 & 63,7 \\
& MUCHO & 2 & 18,1 & 18,1 & 81,8 \\
& MEDIANAMENTE & 1 & 9,1 & 9,1 & 90,9 \\
POCO & 1 & 9,1 & 9,1 & 9,1 \\
NADA & 0 & 0,0 & 0,0 & \\
Total & 11 & 100 & 100 & \\
\hline
\end{tabular}

Fuente: Sistema SPSS 21.

La tabla 5 muestra que un 63,7 \% de los encuestados hacen un amplio uso de la publicidad radial para la promoción de sus campañas electorales; un 18,1 \% indicaron que hay mucho uso; un 9.1 $\%$ medianamente; el $9.1 \%$ poco; nada con $0 \%$ pues no refleja respuestas.

\section{Tabla 6}

¿Utiliza publicidad en medios de prensa para promover su campaña electoral?

\begin{tabular}{llcccc}
\hline & Frecuencia & Porcentaje & $\begin{array}{c}\text { Porcentaje } \\
\text { valido }\end{array}$ & $\begin{array}{c}\text { Porcentaje } \\
\text { acumulado }\end{array}$ \\
\hline Valido & AMPLIAMENTE & 8 & 72,7 & 72,7 & 72,7 \\
& MUCHO & 1 & 9,1 & 9,1 & 81,8 \\
MEDIANAMENTE & 1 & 9,1 & 9,1 & 90,9 \\
POCO & 1 & 9,1 & 9,1 & 9,1 \\
NADA & 0 & 0,0 & 0,0 & \\
Total & 11 & 100 & 100 & \\
\hline
\end{tabular}

Fuente: Sistema SPSS 21.

La tabla 6 despliega que un 72,7 \% de los consultados utilizan ampliamente la publicidad en medios de prensa para la promoción de sus campañas electorales; el 9,1\% expreso que hay mucho uso; un $9.1 \%$ medianamente; el $9.1 \%$ poco; nada con $0 \%$ de contestaciones. 


\section{Tabla 7}

¿Utiliza publicidad en medios impresos (vallas, poster, otros) para promover su campaña electoral?

\begin{tabular}{llcccc}
\hline & Frecuencia & Porcentaje & $\begin{array}{c}\text { Porcentaje } \\
\text { valido }\end{array}$ & $\begin{array}{c}\text { Porcentaje } \\
\text { acumulado }\end{array}$ \\
\hline Valido & AMPLIAMENTE & 9 & 81,8 & 81,8 & 81,8 \\
& MUCHO & 2 & 18,2 & 18,2 & \\
& MEDIANAMENTE & 0 & 0,0 & 0,0 & \\
POCO & 0 & 0,0 & 0,0 & \\
NADA & 0 & 0,0 & 0,0 & \\
$\quad$ Total & 11 & 100 & 100 & \\
\hline
\end{tabular}

Fuente: Sistema SPSS 21.

La tabla 7 señala que un $81,8 \%$ de los precandidatos recurren de forma ampliada a la publicidad por medios impresos para promocionar sus campañas electivas; el 18,2 \% manifiesta que hay mucha utilización de medios y material impreso; no se pronunciaron por las opciones medianamente, poco y nada, es decir datos de $0 \%$ en estas alternativas de respuesta.

\section{Tabla 8}

\section{¿Utiliza para su promoción de campaña electoral la red social Facebook?}

\begin{tabular}{llcccc}
\hline & Frecuencia & Porcentaje & $\begin{array}{c}\text { Porcentaje } \\
\text { valido }\end{array}$ & $\begin{array}{c}\text { Porcentaje } \\
\text { acumulado }\end{array}$ \\
\hline Valido & AMPLIAMENTE & 3 & 27,3 & 27,3 & 27,3 \\
& MUCHO & 4 & 36,4 & 36,4 & 63,7 \\
& MEDIANAMENTE & 1 & 9,1 & 9,1 & 72,8 \\
& POCO & 2 & 18,1 & 18,1 & 90,9 \\
NADA & 1 & 9,1 & 9,1 & \\
Total & 11 & 100 & 100 & \\
\hline
\end{tabular}

Fuente: Sistema SPSS 21.

La tabla 8 deja ver que un 27,3\% de los precandidatos encuestados utilizan ampliamente la red social Facebook para promocionar sus campañas electorales; el 36,4 \% manifiesto darle mucho uso; un $9.1 \%$ medianamente; $18.1 \%$ poco y nada con $9.1 \%$. 
Tabla 9

¿Utiliza para su promoción de campaña electoral la red social Twitter?

\begin{tabular}{llcccc}
\hline & Frecuencia & Porcentaje & $\begin{array}{c}\text { Porcentaje } \\
\text { valido }\end{array}$ & $\begin{array}{c}\text { Porcentaje } \\
\text { acumulado }\end{array}$ \\
\hline Valido & AMPLIAMENTE & 4 & 36,4 & 36,4 & 36.4 \\
& MUCHO & 3 & 27,3 & 27,3 & 63,7 \\
& MEDIANAMENTE & 2 & 18,1 & 18,1 & 81,8 \\
& POCO & 1 & 9,1 & 9,1 & 90,9 \\
& NADA & 1 & 9,1 & 9,1 & \\
Total & 11 & 100 & 100 & \\
\end{tabular}

Fuente: Sistema SPSS 21.

La tabla 9 expone que un 36,4\% de los precandidatos emplean ampliamente la red social Twitter para impulsar promocionalmente sus campañas; el 27,3 \% señala darle mucho uso; un $18.2 \%$ lo hace medianamente; $9.1 \%$ poco y nada con $9.1 \%$.

Tabla 10

¿Utiliza para su promoción de campaña electoral la red social Instagram?

\begin{tabular}{llcccc}
\hline & Frecuencia & Porcentaje & $\begin{array}{c}\text { Porcentaje } \\
\text { valido }\end{array}$ & $\begin{array}{c}\text { Porcentaje } \\
\text { acumulado }\end{array}$ \\
\hline Valido & AMPLIAMENTE & 3 & 27,3 & 27,3 & 27.3 \\
& MUCHO & 1 & 9,1 & 9,1 & 36,4 \\
& MEDIANAMENTE & 4 & 36,4 & 36,4 & 72,8 \\
& POCO & 2 & 18,1 & 18,1 & 90,9 \\
NADA & 1 & 9,1 & 9,1 & \\
Total & 11 & 100 & 100 &
\end{tabular}

Fuente: Sistema SPSS 21.

La tabla 10 explica que un 27,3\% de los precandidatos emplean ampliamente la red social Instagram para promover sus campañas; el 9,1\% enuncia darle mucho uso; un $36.4 \%$ lo utiliza medianamente; $18.1 \%$ poco y un $9.1 \%$ nada. 


\section{Tabla 11}

\section{¿Utiliza alguna página Web para la promoción de su campaña electoral?}

\begin{tabular}{llcccc}
\hline & Frecuencia & Porcentaje & $\begin{array}{c}\text { Porcentaje } \\
\text { valido }\end{array}$ & $\begin{array}{c}\text { Porcentaje } \\
\text { acumulado }\end{array}$ \\
\hline Valido & AMPLIAMENTE & 4 & 36,4 & 36,4 & 36.4 \\
& MUCHO & 2 & 18,1 & 18,1 & 54,5 \\
& MEDIANAMENTE & 1 & 9,1 & 9,1 & 63,6 \\
POCO & 3 & 27,3 & 27,3 & 90,9 \\
NADA & 1 & 9,1 & 9,1 & \\
Total & 11 & 100 & 100 &
\end{tabular}

Fuente: Sistema SPSS 21.

En la tabla 11 se puede apreciar que un $36,4 \%$ de los precandidatos aprovechan ampliamente el uso de una página Web para avivar sus campañas; un $18,1 \%$ se pronuncia por darle mucho uso; un $9.1 \%$ lo utiliza medianamente; el $27.3 \%$ poco y nada un $9.1 \%$.

\section{Tabla 12}

¿Sabe cómo lograr un buen posicionamiento en la mente de los votantes?

\begin{tabular}{llcccc}
\hline & Frecuencia & Porcentaje & $\begin{array}{c}\text { Porcentaje } \\
\text { valido }\end{array}$ & $\begin{array}{c}\text { Porcentaje } \\
\text { acumulado }\end{array}$ \\
\hline Valido & AMPLIAMENTE & 4 & 36,4 & 36,4 & 36.4 \\
& MUCHO & 1 & 9,1 & 9,1 & 45,5 \\
MEDIANAMENTE & 2 & 18,1 & 18,1 & 63,6 \\
POCO & 3 & 27,3 & 27,3 & 90,9 \\
NADA & 1 & 9,1 & 9,1 & \\
Total & 11 & 100 & 100 &
\end{tabular}

Fuente: Sistema SPSS 21.

El registro de la tabla 12 expone que un $36,4 \%$ de los precandidatos saben ampliamente como lograr un buen posicionamiento en la mente de los electores; un 9,1\% se pronuncia por conocer mucho de esta tarea; un $18.1 \%$ medianamente; el $27.3 \%$ poco y un $9.1 \%$ nada. 
Tabla 13

¿Cuenta con una planificación para su campaña?

\begin{tabular}{llcccc}
\hline & Frecuencia & Porcentaje & $\begin{array}{c}\text { Porcentaje } \\
\text { valido }\end{array}$ & $\begin{array}{c}\text { Porcentaje } \\
\text { acumulado }\end{array}$ \\
\hline Valido & AMPLIAMENTE & 4 & 36,4 & 36,4 & 36.4 \\
& MUCHO & 3 & 27,3 & 27,3 & 67,3 \\
& MEDIANAMENTE & 1 & 9,1 & 9,1 & 72,8 \\
& POCO & 1 & 9,1 & 9,1 & 81,9 \\
NADA & 2 & 18,1 & 18,1 & \\
Total & 11 & 100 & 100 & \\
\hline
\end{tabular}

Fuente: Sistema SPSS 21.

La tabla 13 exhibe que un $36,4 \%$ de los precandidatos cuentan ampliamente con una planificación para su campaña; el 27,3\% tomo la opción mucho; un $9.1 \%$ medianamente, otro $9.1 \%$ por la alternativa poco y un $18.1 \%$ se tabulo por el nada.

\section{Tabla 14}

¿Ha desarrollado algún logotipo para identificar su campaña?

\begin{tabular}{llcccc}
\hline & Frecuencia & Porcentaje & $\begin{array}{c}\text { Porcentaje } \\
\text { valido }\end{array}$ & $\begin{array}{c}\text { Porcentaje } \\
\text { acumulado }\end{array}$ \\
\hline Valido & AMPLIAMENTE & 4 & 36,4 & 36,4 & 36.4 \\
& MUCHO & 3 & 27,3 & 27,3 & 67,3 \\
& MEDIANAMENTE & 2 & 18,1 & 18,1 & 81,8 \\
& POCO & 1 & 9,1 & 9,1 & 90,9 \\
NADA & 1 & 9,1 & 9,1 & \\
Total & 11 & 100 & 100 & \\
\hline
\end{tabular}

Fuente: Sistema SPSS 21.

La presente tabla 14, presenta un $36,4 \%$ por la alternativa ampliamente, respecto al desarrollo de un logotipo para identificar su campaña; un 27,3\% se expresó por la opción mucho; un $18.1 \%$ dijo que medianamente, el $9.1 \%$ por la opción poco y otro $9.1 \%$ optó por el nada. 


\section{Tabla 15}

\section{¿Ha creado un slogan de campaña?}

\begin{tabular}{llcccc}
\hline & Frecuencia & Porcentaje & $\begin{array}{c}\text { Porcentaje } \\
\text { valido }\end{array}$ & $\begin{array}{c}\text { Porcentaje } \\
\text { acumulado }\end{array}$ \\
\hline Valido & AMPLIAMENTE & 4 & 36,4 & 36,4 & 36.4 \\
& MUCHO & 2 & 18,1 & 18,1 & 54,5 \\
& MEDIANAMENTE & 3 & 27,3 & 27,3 & 81,8 \\
& POCO & 1 & 9,1 & 9,1 & 90,9 \\
& NADA & 1 & 9,1 & 9,1 & \\
Total & 11 & 100 & 100 & \\
\hline
\end{tabular}

Fuente: Sistema SPSS 21.

El resultado de la tabla 15 indica que un 36,4\% declaro la alternativa ampliamente, respecto a la creación de un slogan para su campaña; el 18,1\% se pronunció por la opción mucho; un $27.3 \%$ señaló que medianamente; un $9.1 \%$ por la opción poco y otro $9.1 \%$ por el nada.

\section{Tabla 16}

\section{¿Controla los avances de su campaña?}

\begin{tabular}{llcccc}
\hline \multirow{2}{*}{ Valido } & Frecuencia & Porcentaje & $\begin{array}{c}\text { Porcentaje } \\
\text { valido }\end{array}$ & $\begin{array}{c}\text { Porcentaje } \\
\text { acumulado }\end{array}$ \\
& AMPLIAMENTE & 4 & 36,4 & 36,4 & 36.4 \\
& MUCHO & 2 & 18,1 & 18,1 & 54,5 \\
MEDIANAMENTE & 3 & 27,3 & 27,3 & 81,8 \\
POCO & 1 & 9,1 & 9,1 & 90,9 \\
NADA & 1 & 9,1 & 9,1 & \\
Total & 11 & 100 & 100 & \\
\hline
\end{tabular}

Fuente: Sistema SPSS 21.

A través de la tabla 16 se conoce que un $36,4 \%$ se pronunció por la opción ampliamente; el 18,1 $\%$ se manifestó por la alternativa mucho; $27.3 \%$ señalaron que medianamente; el $9.1 \%$ dijeron que poco, mientras que un $9.1 \%$ se expresó por el nada. 
Tabla 17

¿Desarrolla sondeos de opinión?

\begin{tabular}{llcccc}
\hline & Frecuencia & Porcentaje & $\begin{array}{c}\text { Porcentaje } \\
\text { valido }\end{array}$ & $\begin{array}{c}\text { Porcentaje } \\
\text { acumulado }\end{array}$ \\
\hline Valido & AMPLIAMENTE & 9 & 81,8 & 81,8 & 81.8 \\
& MUCHO & 1 & 9,1 & 9,1 & 90,9 \\
& MEDIANAMENTE & 0 & 0,0 & 0,0 & 90.9 \\
& POCO & 1 & 9,1 & 9,1 & \\
NADA & 0 & 0,0 & 0,0 & \\
Total & 11 & 100 & 100 & \\
\hline
\end{tabular}

Fuente: Sistema SPSS 21.

Los resultados de la tabla 17 hacen conocer que un $81,8 \%$ de los precandidatos utilizan ampliamente los sondeos de opinión; un 9,1 \% indican mucho uso; por la opción medianamente no hubo respuestas; por la alternativa poco hubo un $9.1 \%$, finalmente un $9.1 \%$ señalo que nada.

Tabla 18

¿Utiliza Tracking Poll?

\begin{tabular}{llcccc}
\hline & Frecuencia & Porcentaje & $\begin{array}{c}\text { Porcentaje } \\
\text { valido }\end{array}$ & $\begin{array}{c}\text { Porcentaje } \\
\text { acumulado }\end{array}$ \\
\hline Valido & AMPLIAMENTE & 2 & 18,1 & 18,1 & 18.1 \\
& MUCHO & 1 & 9,1 & 9,1 & 27,2 \\
& MEDIANAMENTE & 4 & 36,4 & 36,4 & 63.6 \\
& POCO & 1 & 9,1 & 9,1 & 72,7 \\
NADA & 3 & 27,3 & 27,3 & \\
Total & 11 & 100 & 100 & \\
\hline
\end{tabular}

Fuente: Sistema SPSS 21.

La presente tabla 18 permite observar que un $18,1 \%$ de los encuestados manejan ampliamente la herramienta del Tracking Poll; un 9,1\% revelan que mucho; 36,4 \% medianamente; $9.1 \%$ poco y un $27,3 \%$ indico que nada. 


\section{Tabla 19}

\section{¿Hace seguimiento a las opiniones de prensa?}

\begin{tabular}{llcccc}
\hline & Frecuencia & Porcentaje & $\begin{array}{c}\text { Porcentaje } \\
\text { valido }\end{array}$ & $\begin{array}{c}\text { Porcentaje } \\
\text { acumulado }\end{array}$ \\
\hline Valido & AMPLIAMENTE & 7 & 63,7 & 63,7 & 63,7 \\
& MUCHO & 2 & 18,1 & 18,1 & 81,8 \\
& MEDIANAMENTE & 1 & 9,1 & 9,1 & 90,9 \\
POCO & 1 & 9,1 & 9,1 & \\
NADA & 0 & 0,0 & 0,0 & \\
Total & 11 & 100 & 100 & \\
\hline
\end{tabular}

Fuente: Sistema SPSS 21.

En la tabla 19 se puede evidenciar que un 63,7\% de los encuestados, realizan de forma amplia el seguimiento a las opiniones de la prensa; el 18,1 \% también lo hace con mucha frecuencia; el 9,1 $\%$ lo realiza medianamente, mientras que un $9,1 \%$ tiene poca práctica en este hábito de control; por la opción nada no existen respuestas.

\section{Tabla 20}

¿Escucha u observa opiniones especializadas en política?

\begin{tabular}{llcccc}
\hline \multirow{2}{*}{ Valido } & Frecuencia & Porcentaje & $\begin{array}{c}\text { Porcentaje } \\
\text { valido }\end{array}$ & $\begin{array}{c}\text { Porcentaje } \\
\text { acumulado }\end{array}$ \\
& AMPLIAMENTE & 7 & 63,7 & 63,7 & 63,7 \\
& MUCHO & 2 & 18,1 & 18,1 & 81,8 \\
MEDIANAMENTE & 1 & 9,1 & 9,1 & 90,9 \\
POCO & 1 & 9,1 & 9,1 & \\
NADA & 0 & 0,0 & 0,0 & \\
Total & 11 & 100 & 100 & \\
\hline
\end{tabular}

Fuente: Sistema SPSS 21.

Tras el resultado que refleja la tabla 20 se observa que el $63,7 \%$ de los consultados escuchan u observan ampliamente las opiniones especializadas en política; un 18,1 \% lo efectúa con mucha 
continuidad; un 9,1\% lo ejecuta medianamente; otro 9,1\% tiene poca costumbre de hacerlo; la opción nada no presenta contestaciones.

Tabla 21

¿Se apoya con asesores y equipo de trabajo?

\begin{tabular}{llcccc}
\hline & Frecuencia & Porcentaje & $\begin{array}{c}\text { Porcentaje } \\
\text { valido }\end{array}$ & $\begin{array}{c}\text { Porcentaje } \\
\text { acumulado }\end{array}$ \\
\hline Valido & AMPLIAMENTE & 8 & 72,8 & 72,8 & 72,8 \\
& MUCHO & 2 & 18,1 & 18,1 & 90,9 \\
& MEDIANAMENTE & 0 & 0,0 & 0,0 & \\
POCO & 1 & 9,1 & 9,1 & \\
NADA & 0 & 0,0 & 0,0 & \\
Total & 11 & 100 & 100 & \\
\hline
\end{tabular}

Fuente: Sistema SPSS 21.

La tabla 21 deja ver que el 72,8\% de los encuestados se apoyan de manera amplia con asesores y equipo de trabajo; $18,1 \%$ de los consultados indican hacerlo con mucha frecuencia; la opción medianamente no refleja respuestas; un $9,1 \%$ lo hace muy poco; finalmente por la alternativa nada no se generaron respuestas.

\section{Tabla 22}

¿Las herramientas de Marketing Político han logrado darle resultados?

\begin{tabular}{llcccc}
\hline & Frecuencia & Porcentaje & $\begin{array}{c}\text { Porcentaje } \\
\text { valido }\end{array}$ & $\begin{array}{c}\text { Porcentaje } \\
\text { acumulado }\end{array}$ \\
\hline Valido & AMPLIAMENTE & 7 & 63,8 & 63,8 & 63,8 \\
& MUCHO & 2 & 18,1 & 18,1 & 81,9 \\
MEDIANAMENTE & 2 & 18,1 & 18,1 & \\
POCO & 0 & 0.0 & 0,0 & \\
NADA & 0 & 0,0 & 0,0 & \\
Total & 11 & 100 & 100 &
\end{tabular}

Fuente: Sistema SPSS 21. 
Es visible de acuerdo a la tabla 22 que el 63,8 \% de los precandidatos encuestados, han logrado ampliamente darles mejores resultados a sus campañas gracias a la utilización de herramientas de Marketing Político; el 18,1 \% señalan haber logrado muchos y mejores resultados; por la alternativa medianamente respondieron el 9,1\%, mientras que por las opciones poco y nada no se reportaron respuestas.

\section{Discusión y conclusiones}

En Ecuador y América Latina en particular, por raíces e idiosincrasias parecidas, los electores no sufragan de forma racional, pues sus decisiones a la hora de votar las basan en el sentimentalismo y emociones, como por ejemplo la esperanza, el amor, odio, miedo, interés y otras sensibilidades. Precisamente el abordaje de esta investigación es saber cuánto conocen y manejan los precandidatos a la Alcaldía de Manta el Marketing Político (MP), el cual brinda un conjunto de métodos, técnicas y herramientas que hacen posible descifrar las necesidades de los votantes o pobladores, transformándose en una opción diferente para realizar una campaña electoral, pues predomina $100 \%$ el factor estratégico.

Es así que el MP es el nuevo estilo de hacer política, donde tanto el partido como los grupos de interés cobran gran importancia, por lo que la actividad del candidato debe ser cotidiana y permanente, dado que las organizaciones políticas apuestan a ganar. La mercadotecnia, contribuye a la generación de una comunicación abierta, de tipo horizontal entre el postulante y el votante, para ello las TICs, a través de los medios masivos como las redes sociales, son quizá el instrumento más valioso que tiene un político; hoy es posible minimizar el mal diseño de una estrategia y táctica política, por ello es importante citar a Joseph Napolitan, quien señala que "una estrategia correcta puede sobrevivir a una campaña mediocre, pero una campana brillante puede fracasar si la estrategia está equivocada”, he aquí la relevancia del Marketing Político.

Los resultados del presente estudio, visto desde la validación de la hipótesis investigativa, permitieron a través del uso del programa estadístico SPSS 21 establecer que: El Marketing Político impacta en la campaña electoral de los precandidatos a la Alcaldía de Manta - Ecuador, con un Rho de Spearman de 0,964 evidenciando un nivel de correlación positiva significativa o alta, con un nivel de confiabilidad cercano a 1; se reitera entonces que a mayor nivel de aplicación de marketing político mayor será el impacto en la campaña electoral de un candidato. 
Este trabajo también cumplió con el objetivo trazado, cual fue: Determinar de qué manera el Marketing Político impacta en la campaña electoral de los precandidatos a la Alcaldía de Manta Ecuador.

De los resultados generales, se aprecia que la mayoría de los precandidatos aprovechan los medios masivos como las redes sociales para difundir sus propuestas, y eso es más evidente en los postulantes jóvenes, los cuales tienen una conexión más fluida y directa con el elector coetáneo.

\section{Referencias Bibliográficas}

Avraham Shama (1981). "Marketing Solar Energy Innovations”. Ed. Kindle.

Campbell y Stanley (1978). “Diseños experimentales y cuasi experimentales en la investigación social". Amorrourtu Editores.

Carmen Fernández \& Luz Reyes (2003). “Marketing político: Herramientas para ganar elecciones. Ed. Konrad A.S.

Carmen Fernández \& Luz Reyes (2010). ¿Cómo ganar una elección? Secretos de Marketing político. Ed. El nacional.

Consejo Nacional Electoral (2018). "Inscritos a la Alcaldía del Cantón Manta”. CNE.

Daniel Skibel (2015). "Maquiavelo y Freud: Psicología política para ganar elecciones”. Ed.BGP.

John W. Creswell (2008). “Investigación cualitativa y diseño investigativo”. SAGE. Publications. Josehp Napolitan (2011). "10 andamientos de una campaña política”. Ed. McGraw Hill.

Juan R. Renedo (2002). "Neurociencia en la política. Ed. Business Books.

Lourdes M. Salgado (2002). "Marketing político, arte y ciencia de la persuasión en democracia. Ed. Paidós.

Luis C. Bonino (2005). “Manual de Marketing político”. Ed. Fin de siglo.

Philippe J. Maarek (1997). “Marketing político y comunicación: Claves para una buena información política. Ed. Paidós. España.

Wojciech Cwalina, Andrzej Falkoski y Bruce I. Newman, (2015). "Political Marketing: Theoretical and Strategic Foudations". Ed. Routledge. 